\title{
ELECTRON STATES AND ADIABATIC POTENTIAL OF THE HYDROGEN BOND
}

\author{
I. V. Stasyuk, Yu. V. Sizonenko, R. Ya. Stetsiv \\ Institute for Condensed Matter Physics of the National Academy of Sciences of Ukraine \\ 1 Svientsitskii Str., Lviv, UA-290011, Ukraine
}

(Received February 3, 1998; received in final form May 8, 1998)

\begin{abstract}
The properties of the hydrogen bond are investigated on the basis of the simplest object with hydrogen bond, the ionic complex $\mathrm{O}-\mathrm{H}-\mathrm{O}$. The electron energy spectrum of the $\mathrm{O}-\mathrm{H}-\mathrm{O}$ complex is obtained. The formation of the two-minima adiabatic proton potential as a function of the hydrogen bond length $R_{\odot \odot}, R_{\odot \mathrm{H}}$ distance, electron number $N$ in complex is investigated. The electron density maps and the distribution of the electron charge along the complex axis are obtained. We have also studied the change of the electron charge density and the occupancy of the atomic orbitals of the initial basis connected with proton shift along the hydrogen bond.
\end{abstract}

Key words: hydrogen bond, adiabatic potential, exchange integral, electron states.

PACS number(s): 31.25.Qm, 36.40.-C

\section{INTRODUCTION}

There are many papers devoted to the investigation of the hydrogen bond systems including review papers shedding some light on the nature of the hydrogen bond [1-5]. The main subjects are the study of the electron structure, optimum geometry, investigation of the adiabatic potentials for the proton, vibrational spectra, proton transfer. Nevertheless, the problem of the formation of adiabatic potential for the proton calls for a more detailed study. For example the role of the Coulomb components of the interaction between excessive charges, which appear during the displacement of ions and electron redistribution, are not sufficiently studied. In paper [6] the authors propose new model in which a strong coupling between the protons and distortion of the structural units, connected by the hydrogen bonds (for example, $\mathrm{PO}_{4}$ tetrahedrons in $\mathrm{KH}_{2} \mathrm{PO}_{4}$ crystal) is assumed and the interaction between protons is disregarded. It is supposed there that the distortion of tetrahedron is proportional to its electric dipole moment. One should try to extend such models, as not only dipole moment on the edge structure units changes with the proton motions along hydrogen bond, but the charge value is changed also.

The data, obtained recently while investigating complexes with the hydrogen bonds $\left\{M A_{2} X\right\} Y_{2}$, (where $M=\mathrm{Pt}, \mathrm{Pd}$ or $\mathrm{Ni} ; X=\mathrm{Cl}, \mathrm{Br}$ or $\mathrm{J} ; Y=\mathrm{Br}^{-}, \mathrm{ClO}_{4}^{-} ; A-$ ligand molecule) shows us the importance of the consideration of the electron charge redistribution which accompanies proton shifts on the bond [7]. In this case it was established that additional electron charges (at some conditions they form the charge-ordered state) are inducted on the ions $M$ depending on the location of protons on $N-H-Y$. Electron redistribution, however, plays on important role at the formation of locally anharmonic potentials in the lattice. This conclusion follows from the investigations, performed in the frames of the cluster approach with consideration of the electron- vibrational interactions (see $[8,9]$ ).

The development of rather simple models, that describes such a situation, calls for a more detailed study of the phenomena connected with the electron transfer on the hydrogen bonds. In the present paper this problem is analyzed on the basis of the quantum-chemical calculations.

We consider the ionic complex $\mathrm{O}-\mathrm{H}-\mathrm{O}$ (further on referred to as $A-B-A^{\prime}$ ). The Hamiltonian of such a complex has the following form:

$$
\begin{aligned}
H(\mathbf{r}, R) & =\sum_{i}\left(-\frac{\hbar^{2}}{2 m} \Delta_{i}\right)+\sum_{i<j} \frac{e^{2}}{\left|\mathbf{r}_{i}-\mathbf{r}_{j}\right|}+\sum_{i, n} U\left(\mathbf{r}_{i}-\mathbf{R}_{n}\right) \\
& +\sum_{n}\left(-\frac{\hbar^{2}}{2 M_{n}} \Delta_{n}\right)+\sum_{m, n}^{\prime} W\left(\mathbf{R}_{m}-\mathbf{R}_{n}\right)
\end{aligned}
$$

where $\mathbf{r}_{i}, \mathbf{R}_{m}$ - electron and ion coordinates.

The first three terms form the electron part of the Hamiltonian; the last two describe the ionic subsystem;

$$
\begin{aligned}
& U\left(\mathbf{r}_{i}-\mathbf{R}_{n}\right)=\frac{e^{2} Z_{e l}}{\left|\mathbf{r}_{i}-\mathbf{R}_{n}\right|}, \\
& W\left(\mathbf{R}_{m}-\mathbf{R}_{n}\right)=\frac{e^{2} Z_{i o n}}{\left|\mathbf{R}_{m}-\mathbf{R}_{n}\right|}
\end{aligned}
$$

here $Z_{e l}$ and $Z_{i o n}$ are the effective ion charges (excluding valence electrons) used for the description of the interaction between them and other electrons or ions respectively.

In the present paper we calculate the electron spectrum of the $\mathrm{O}-\mathrm{H}-\mathrm{O}$ cluster with taking into account the electron transfer, Coulomb and exchange interactions. The formation of the adiabatic proton potential as a function of the hydrogen bond length $R_{\mathrm{OO}}, R_{\mathrm{OH}}$ distance, electron number in the cluster $N$ is investigated. 
The electron density pictures and distribution of the electron charge along the complex axis is obtained. We investigate also a change of the electron charge density with the proton shift along the bond.

\section{INITIAL BASIS OF ELECTRON WAVE FUNCTIONS AND ITS ORTHOGONALIZATION.}

In our model approach we take into consideration the minimum basis of electron wave functions consisting of the three valence atomic orbitals. The $2 p$-functions of the oxygen ions $\left(\left\{\psi_{a}\left(\mathbf{r}-\mathbf{R}_{a}\right)\right\},\left\{\psi_{a^{\prime}}\left(\mathbf{r}-\mathbf{R}_{a^{\prime}}\right)\right\}\right)$, directed along the hydrogen bond and $1 s$-function of the hydrogen atom $\left(\left\{\psi_{b}\left(\mathbf{r}-\mathbf{R}_{b}\right)\right\}\right)$ are taken into account.

Using the $s p^{3}$ orbitals on the oxygen ions as the basis surely will be more correct, however, we suppose, that the error caused by our simple approximation, can be minimized by the appropriate choice of the $Z_{e l}$ and $Z_{i o n}$ parameters. The basis functions are the solutions of the Schrödinger equations

$$
\left[\sum_{i}\left(-\frac{\hbar^{2}}{2 m} \Delta_{i}\right)+U\left(\mathbf{r}_{i}-\mathbf{R}_{s}\right)\right] \psi_{s}\left(\mathbf{r}_{i}\right)=E_{s} \psi_{s}\left(\mathbf{r}_{i}\right)
$$

(here $s=a, b, a^{\prime}$ ).

At first the orthogonalization of the functions $\psi_{a}$ and $\psi_{a^{\prime}}$ is performed:

$$
\begin{gathered}
\tilde{\psi_{a}}=N_{1}\left(\psi_{a}+\psi_{a^{\prime}}\right), N_{1}=\frac{1}{\sqrt{2\left(1+S_{a a^{\prime}}\right)}}, \\
\tilde{\psi_{a^{\prime}}}=N_{2}\left(\psi_{a}-\psi_{a^{\prime}}\right), N_{2}=\frac{1}{\sqrt{2\left(1-S_{a a^{\prime}}\right)}} .
\end{gathered}
$$

Here $S_{a a^{\prime}}=\int \psi_{a}^{*}(\mathbf{r}) \psi_{a^{\prime}}(\mathbf{r}) d \mathbf{r}$ - overlap integral of the initial atomic function.

The transformation of the vector $45^{\circ}$ rotation type is used for the symmetrization of the new basis:

$$
\begin{aligned}
& \varphi_{a}(\mathbf{r})=\frac{1}{\sqrt{2}}\left(\tilde{\psi}_{a}+\tilde{\psi_{a^{\prime}}}\right)=\xi \psi\left(\mathbf{r}-\mathbf{R}_{a}\right)+\eta \psi\left(\mathbf{r}-\mathbf{R}_{a^{\prime}}\right) \\
& \varphi_{a^{\prime}}(\mathbf{r})=\frac{1}{\sqrt{2}}\left(\tilde{\psi_{a}}-\tilde{\psi}_{a^{\prime}}\right)=\eta \psi\left(\mathbf{r}-\mathbf{R}_{a}\right)+\xi \psi\left(\mathbf{r}-\mathbf{R}_{a^{\prime}}\right) .
\end{aligned}
$$

Here

$$
\begin{aligned}
& \xi=\frac{1}{2 \sqrt{\left(1+S_{a a^{\prime}}\right)}}+\frac{1}{2 \sqrt{\left(1-S_{a a^{\prime}}\right)}}, \\
& \eta=\frac{1}{2 \sqrt{\left(1+S_{a a^{\prime}}\right)}}-\frac{1}{2 \sqrt{\left(1-S_{a a^{\prime}}\right)}} .
\end{aligned}
$$

$$
\epsilon_{1}=t_{a a} ; \quad \epsilon_{2}=t_{b b} ; \quad \epsilon_{3}=t_{a^{\prime} a^{\prime}} ; \quad t=t_{a b} ; \quad t^{\prime}=t_{a^{\prime} b} ;
$$

Orthogonalizing the third function to the first and the second ones gives:

$$
\begin{aligned}
\varphi_{b} & =\rho \psi_{b}\left(\mathbf{r}-\mathbf{R}_{b}\right)+\xi_{a} \varphi_{a}+\xi_{a^{\prime}} \varphi_{a^{\prime}} \\
& =\rho \psi\left(\mathbf{r}-\mathbf{R}_{b}\right)+\left(\xi_{a} \xi+\xi_{a^{\prime}} \eta\right) \psi\left(\mathbf{r}-\mathbf{R}_{a}\right) \\
& +\left(\xi_{a^{\prime}} \xi+\xi_{a} \eta\right) \psi\left(\mathbf{r}-\mathbf{R}_{a^{\prime}}\right) .
\end{aligned}
$$

Here the following notations are introduced:

$$
\begin{aligned}
& \rho=\sqrt{\frac{1-S_{a a^{\prime}}^{2}}{1-S_{a a^{\prime}}^{2}-S_{b a}^{2}-S_{b a^{\prime}}^{2}+S_{b a} S_{b a^{\prime}} S_{a a^{\prime}}}} ; \\
& \xi_{a}=-\left(S_{b a} \xi+S_{b a^{\prime}} \eta\right) \rho \\
& \xi_{a^{\prime}}=-\left(S_{b a} \eta+S_{b a^{\prime}} \xi\right) \rho .
\end{aligned}
$$

The functions $\left\{\varphi_{a}, \varphi_{b}, \varphi_{a}^{\prime}\right\}$ form an orthogonalized and normalized basis of states.

\section{ELECTRON SPECTRA OF O-H-O CLUSTER.}

Electron part of the Hamiltonian (1) in secondary quantization representation on the $\left\{\varphi_{l}(\mathbf{r})\right\}$ basis has the following form:

$$
\begin{aligned}
H_{e l}(R) & =\sum_{i, j, \sigma} t_{i, j} c_{i, \sigma}^{\dagger} c_{j, \sigma} \\
& +\frac{1}{2} \sum_{i, j, k, l} \sum_{\sigma, \sigma^{\prime}}\left\langle i j\left|\frac{e^{2}}{\left|\mathbf{r}_{1}-\mathbf{r}_{2}\right|}\right| k l\right\rangle c_{i, \sigma}^{\dagger} c_{j, \sigma^{\prime}}^{\dagger} c_{l, \sigma^{\prime}} c_{k, \sigma} .
\end{aligned}
$$

Here

$$
\begin{aligned}
& t_{i j}=\left\langle i\left|-\frac{\hbar^{2}}{2 m} \Delta+\sum_{m} U\left(\mathbf{r}-\mathbf{R}_{m}\right)\right| j\right\rangle= \\
& =\int \varphi_{i}^{*}(\mathbf{r})\left(-\frac{\hbar^{2}}{2 m} \Delta+\sum_{m} U\left(\mathbf{r}-\mathbf{R}_{m}\right)\right) \varphi_{j}(\mathbf{r}) d \mathbf{r} ; \\
& \langle i j|\ldots| k l\rangle \equiv\left\langle i j\left|\frac{e^{2}}{\left|\mathbf{r}_{1}-\mathbf{r}_{2}\right|}\right| k l\right\rangle= \\
& \int \varphi_{i}^{*}\left(\mathbf{r}_{\mathbf{1}}\right) \varphi_{j}^{*}\left(\mathbf{r}_{\mathbf{2}}\right) \frac{e^{2}}{\left|\mathbf{r}_{1}-\mathbf{r}_{2}\right|} \varphi_{k}\left(\mathbf{r}_{\mathbf{1}}\right) \varphi_{l}\left(\mathbf{r}_{2}\right) d \mathbf{r}_{1} d \mathbf{r}_{2} .
\end{aligned}
$$

Further on the following notations for the matrix elements are used: 


$$
\begin{aligned}
& U=\langle a a|\ldots| a a\rangle ; \quad U^{\prime}=\langle b b|\ldots| b b\rangle ; \\
& \Phi=\langle a a|\ldots| a b\rangle ; \quad \Phi_{1}=\langle b b|\ldots| b a\rangle ; \\
& \Phi^{\prime}=\left\langle a^{\prime} a^{\prime}|\ldots| a^{\prime} b\right\rangle ; \quad \Phi_{1}^{\prime}=\left\langle b b|\ldots| b a^{\prime}\right\rangle ; \\
& \Phi_{a}=\left\langle a a^{\prime}|\ldots| a a\right\rangle=\left\langle a^{\prime} a^{\prime}|\ldots| a^{\prime} a\right\rangle ; \\
& L_{1}=\left\langle a a^{\prime}|\ldots| a b\right\rangle ; \quad L_{1}^{\prime}=\left\langle a^{\prime} a|\ldots| a^{\prime} b\right\rangle ; \quad L_{2}=\left\langle b a^{\prime}|\ldots| b a\right\rangle ; \\
& L_{3}=\left\langle a a|\ldots| a^{\prime} b\right\rangle ; \quad L_{3}^{\prime}=\left\langle a^{\prime} a^{\prime}|\ldots| a b\right\rangle ; \quad L_{4}=\left\langle b b|\ldots| a a^{\prime}\right\rangle ; \\
& K=\langle a b|\ldots| a b\rangle ; \quad K^{\prime}=\left\langle a^{\prime} b|\ldots| a^{\prime} b\right\rangle ; \quad V=\left\langle a a^{\prime}|\ldots| a a^{\prime}\right\rangle ; \\
& Q=\langle a a|\ldots| b b\rangle ; \quad Q^{\prime}=\left\langle a^{\prime} a^{\prime}|\ldots| b b\right\rangle ; \quad Q_{a}=\left\langle a a|\ldots| a^{\prime} a^{\prime}\right\rangle,
\end{aligned}
$$

that describe, correspondingly, the energy of the localized levels, electron transfer $\left(t_{s s}, t_{a b}, t_{a^{\prime} b}\right)$, single-center Coulomb interaction between an electron of the Hubbard type $\left(U\right.$ and $\left.U^{\prime}\right)$, correlated electron transfer $\left(\Phi, \Phi_{1}, \Phi^{\prime}\right.$, $\left.\Phi_{1}^{\prime}, \Phi_{a}, L_{i}, L_{i}^{\prime}\right)$, two-centers Coulomb interactions $(K$, $\left.K^{\prime}, V\right)$ and exchange interactions $\left(Q, Q^{\prime}, Q_{a}\right)$.

Let us go to the basis of the electron states in the occupation number representation $\left|n_{a \uparrow} n_{a \downarrow} n_{b \uparrow} n_{b \downarrow} n_{a^{\prime} \uparrow} n_{a^{\prime} \downarrow}\right\rangle$, which includes 64 states:

\begin{tabular}{|l|l||l|l||l|l|}
\hline & $|p\rangle$ & & $|p\rangle$ & & $|p\rangle$ \\
\hline$|000000\rangle$ & $|1\rangle$ & $|11000\rangle$ & $|23\rangle$ & $|111001\rangle$ & $|45\rangle$ \\
\hline$|100000\rangle$ & $|2\rangle$ & $|110100\rangle$ & $|24\rangle$ & $|110110\rangle$ & $|46\rangle$ \\
\hline$|010000\rangle$ & $|3\rangle$ & $|110010\rangle$ & $|25\rangle$ & $|110101\rangle$ & $|47\rangle$ \\
\hline$|001000\rangle$ & $|4\rangle$ & $|11000\rangle\rangle$ & $|26\rangle$ & $|110011\rangle$ & $|48\rangle$ \\
\hline$|000100\rangle$ & $|5\rangle$ & $|101100\rangle$ & $|27\rangle$ & $|101110\rangle$ & $|49\rangle$ \\
\hline$|000010\rangle$ & $|6\rangle$ & $|101010\rangle$ & $|28\rangle$ & $|101101\rangle$ & $|50\rangle$ \\
\hline$|000001\rangle$ & $|7\rangle$ & $|101001\rangle$ & $|29\rangle$ & $|101011\rangle$ & $|51\rangle$ \\
\hline$|110000\rangle$ & $|8\rangle$ & $|100110\rangle$ & $|30\rangle$ & $|10011\rangle$ & $|52\rangle$ \\
\hline$|101000\rangle$ & $|9\rangle$ & $|100101\rangle$ & $|31\rangle$ & $|011110\rangle$ & $|53\rangle$ \\
\hline$|100100\rangle$ & $10\rangle$ & $|10001\rangle\rangle$ & $|32\rangle$ & $|011101\rangle$ & $|54\rangle$ \\
\hline$|100010\rangle$ & $|11\rangle$ & $|011100\rangle$ & $|33\rangle$ & $|011011\rangle$ & $|55\rangle$ \\
\hline$|100001\rangle$ & $|12\rangle$ & $|011010\rangle$ & $|34\rangle$ & $|010111\rangle$ & $|56\rangle$ \\
\hline$|011000\rangle$ & $13\rangle$ & $|011001\rangle$ & $|35\rangle$ & $|00111\rangle$ & $|57\rangle$ \\
\hline$|010100\rangle$ & $|14\rangle$ & $|010110\rangle$ & $|36\rangle$ & $|111110\rangle$ & $|58\rangle$ \\
\hline$|010010\rangle$ & $15\rangle$ & $|010101\rangle$ & $|37\rangle$ & $|111101\rangle$ & $|59\rangle$ \\
\hline$|010001\rangle$ & $|16\rangle$ & $|010011\rangle$ & $|38\rangle$ & $|111011\rangle$ & $|60\rangle$ \\
\hline$|001100\rangle$ & $|17\rangle$ & $|001110\rangle$ & $|39\rangle$ & $|110111\rangle$ & $|61\rangle$ \\
\hline$|001010\rangle$ & $|18\rangle$ & $|001101\rangle$ & $|40\rangle$ & $|101111\rangle$ & $|62\rangle$ \\
\hline$|001001\rangle$ & $|19\rangle$ & $|001011\rangle$ & $|41\rangle$ & $|011111\rangle$ & $|63\rangle$ \\
\hline$|000110\rangle$ & $|20\rangle$ & $|000111\rangle$ & $|42\rangle$ & $|111111\rangle$ & $|64\rangle$ \\
\hline$|000101\rangle$ & $|21\rangle$ & $|111100\rangle$ & $|43\rangle$ & & \\
\hline$|000011\rangle$ & $|22\rangle$ & $|111010\rangle$ & $|44\rangle$ & & \\
\hline
\end{tabular}

Matrix $H_{i j}(R)$ on this basis consists of independent blocks each of them corresponding to a certain number of electrons $N$. It may be seen using the accepted conceptions of valency that the most probable number of electrons on such an $\mathrm{O}-\mathrm{H}-\mathrm{O}$ cluster (in its three valent states that compose the initial basis and organise the hydrogen bond) is close to four.

The realization of other values of $N$ is also possible when the electron transfer between the ions or ion groups participating in forming different hydrogen bonds is taking place.

In the present paper the cases $N=3, N=4, N=5$ are analyzed.

For the $N=4$ case the corresponding basis of states includes 15 states. Matrix $H_{i j}(R)$ of $(15 \times 15)$ size consists of three blocks: $(3 \times 3)$ - summary spin projection $S^{z}=+1 ;(3 \times 3)$ - summary spin projection $S^{z}=-1$; $(9 \times 9)$ - summary spin projection $S^{z}=0$. The most energetically advantageous case $S^{z}=0$ is realised on the states $|43\rangle,|45\rangle,|46\rangle,|48\rangle,|50\rangle,|52\rangle,|53\rangle,|55\rangle$ and $|57\rangle$

For the $N=3$ case matrix $H_{i j}(R)$ of $(20 \times 20)$ size consists of four blocks: $(1 \times 1)$ - summary spin projection $S^{z}=-3 / 2 ;(1 \times 1)$ - summary spin projection $S^{z}=$ $+3 / 2$; $(9 \times 9)$ - summary spin projection $S^{z}=+1 / 2$; $(9 \times 9)$ - summary spin projection $S^{z}=-1 / 2$. Cases with the opposite spin are equivalent. The $S^{z}=1 / 2$ case is realised on the states $|23\rangle,|25\rangle,|27\rangle,|29\rangle,|30\rangle$, $|32\rangle,|34\rangle,|39\rangle$ and $|41\rangle$.

For the $N=5$ case matrix $H_{i j}(R)$ of $(6 \times 6)$ size consists of two blocks:

$(3 \times 3)$ - summary spin projection $S^{z}=+1 / 2 ;(3 \times 3)$ - summary spin projection $S^{z}=-1 / 2$. $S^{z}=1 / 2$ case is realised on the states $|58\rangle,|60\rangle$ and $|62\rangle$.

The components of matrix $H_{i j}(R)$ blocks corresponding to the above mentioned states are given in Appendix 1.

Matrix elements $H_{i j}(R)$ are formed by the matrix elements (10) on the orthogonalized basis of functions $\varphi_{l}(\mathbf{r})$, which in turn are linear combinations of the corresponding matrix elements built on the initial basis of the atomic functions $\psi_{m}(\mathbf{r})$. Analytical expressions obtained for some of them are given in Appendix 2. A general scheme of calculations is described and formulae for the evaluation of exchange integrals are also presented there. For the rest of the matrix elements numerical evaluation was performed (including evaluation of 3 -center integrals) on the basis of the atomic functions $\psi_{m}(\mathbf{r})$. 


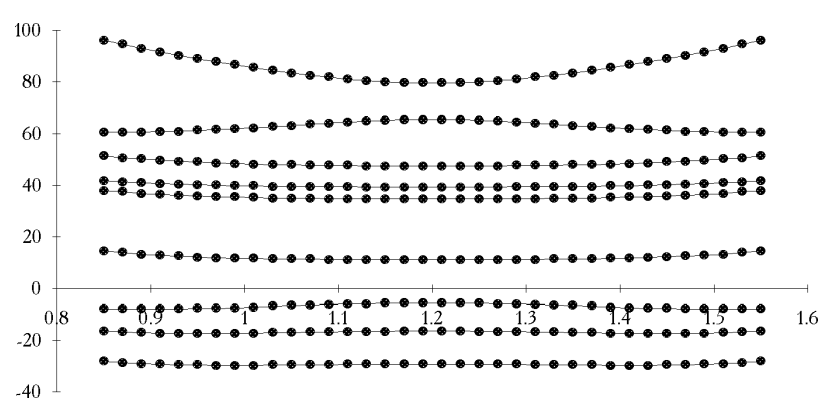

Fig. 1. Dependence of the energy spectrum of the system ( $\mathrm{eV}$ ) on $R_{\mathrm{OH}}$ distance $(\mathrm{A})$ at the following values of parameters: $N=4, R_{\odot \circ}=2.40, Z_{e l}=0.5, Z_{i o n}=4.5$.
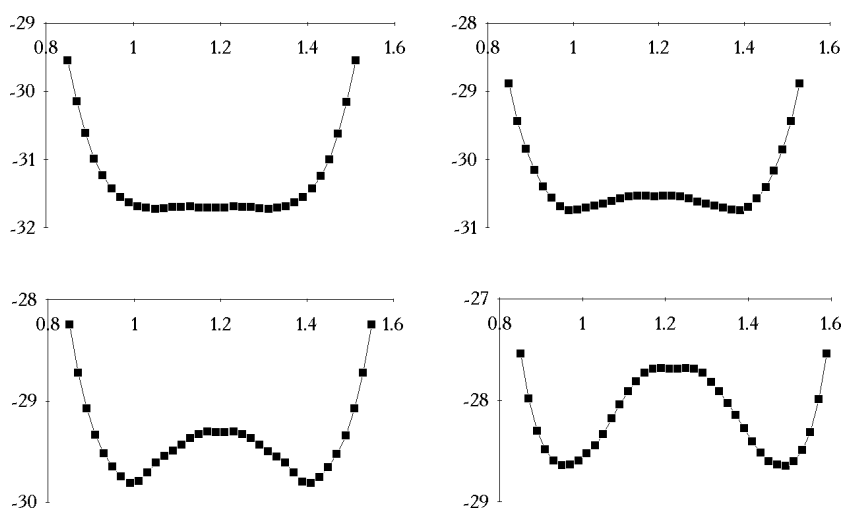

Fig. 2. The lowest level of the system energy spectrum (the proton adiabatic potential) as a function of the $R_{\odot \mathrm{OH}}$ distance at $N=4\left(Z_{e l}=0.5, Z_{i o n}=4.5\right)$ at different values of the bond length: a) $R_{\odot \odot}=2.36 \mathrm{~A}$ c) $R_{\odot \odot}=2.40 \mathrm{~A}$ bond length: b) $\left.R_{\odot \odot}=2.38 \mathrm{~A} \mathrm{~d}\right) R_{\odot \odot}=2.44 \mathrm{~A}$
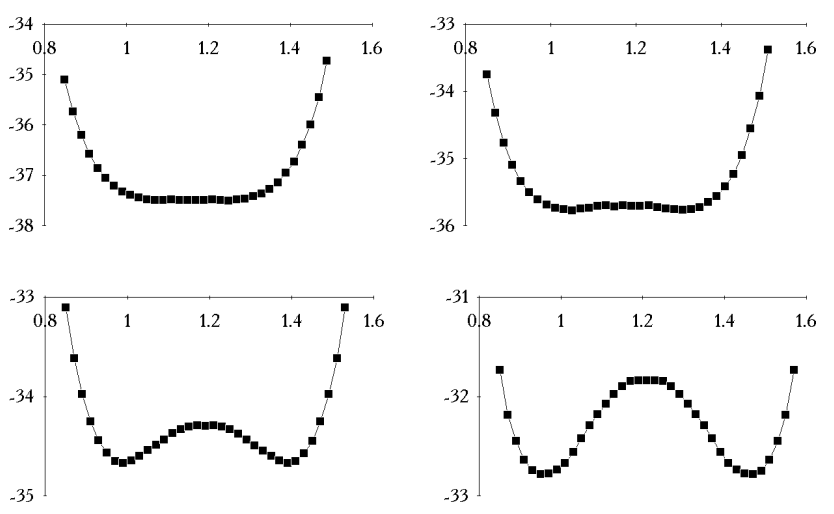

Fig. 3. The lowest level of the energy spectrum of the system (proton adiabatic potential) as a function of the $R_{\mathrm{OH}}$ distance at $N=3\left(Z_{e l}=0.5, Z_{i o n}=4.5\right)$ at different values of the bond length: a) $R_{\odot \circ}=2.33 \mathrm{~A}$ c) $R_{\odot \odot}=2.37 \mathrm{~A}$

b) $R_{\odot \odot}=2.35 \mathrm{~A}$ d) $R_{\odot \odot}=2.40 \mathrm{~A}$
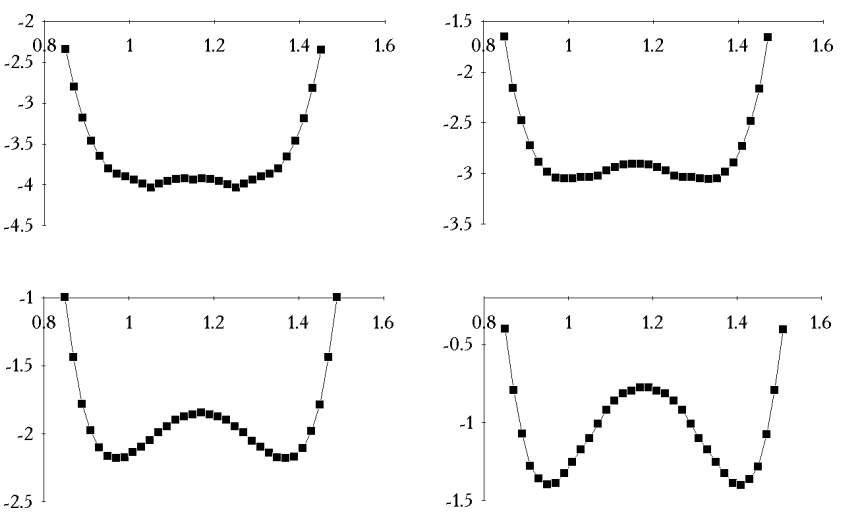

Fig. 4. The lowest level of the energy spectrum of the system (proton adiabatic potential) as a function of the $R_{\mathrm{OH}}$ distance at $N=5\left(Z_{e l}=0.5, Z_{i o n}=4.5\right)$ at different values of the bond length: a) $R_{\odot \odot}=2.30 \mathrm{~A}$ c) $R_{\odot \odot}=2.34 \mathrm{~A}$
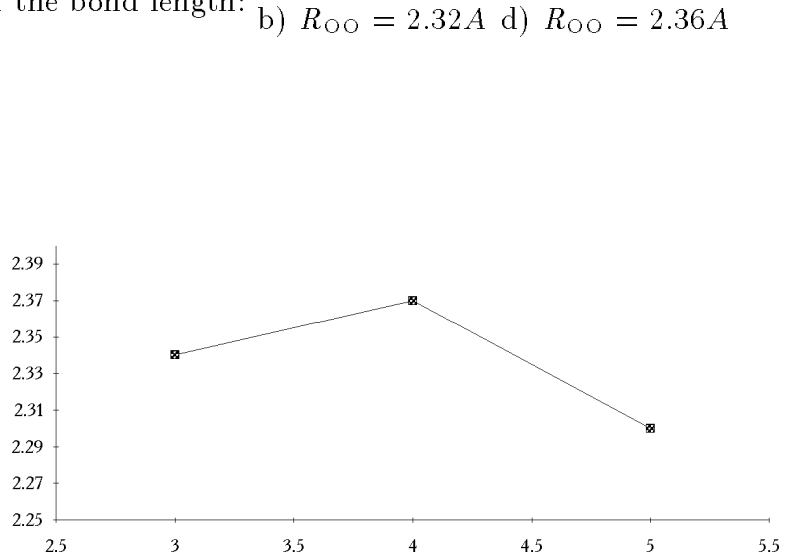

Fig. 5. Dependence of the critical value $R_{\bigcirc \circ}^{c r}$ on the electron number on the complex.

For the numerical calculations the methods of MonteCarlo type for multi-dimensional integrals are used. The error of evaluation was not more than $4 \%$.

A matrix of the Hamiltonian was diagonalized, its eigenvalues $\lambda_{i}$ and eigenvectors were found.

The eigenvalues of the Hamiltonian of the electron subsystem (where the ion-electron and the electron-electron interactions were considered) were added by the energy of the ion-ion interaction (excluding oxygen-oxygen interaction), the result spectrum for the given values of distance $R_{\mathrm{OO}}$ is presented as a dependence on the $R_{\mathrm{OH}}$ distance (see fig. 1).

The lowest level from this spectrum is presented in more details on fig. 2. It may be seen, that at bond length smaller than some critical value, we have a potential with one central minimum, which at the increasing of the complex length transforms into a double-well potential. This fact shows us the existence of two equilibrium states of the system, different in the shift of the central ion in opposite directions along the complex axis. At some values of the effective charges $Z_{e l}$ and $Z_{i o n}$ we can obtain parameters of the proton potential on the hydrogen bond known from the experiments. Optimum $Z$ values are dif- 
ferent for different numbers of electrons on the cluster O$\mathrm{H}-\mathrm{O}$. The results of calculations are especially sensitive to the difference $\Delta Z=Z_{\text {ion }}-Z_{e l}$. The optimal values for the model parameters are $2.0 \leq \alpha \leq 2.1 ; \Delta Z=4.0$ $(N=4) ; \Delta Z=4.3(N=3) ; \Delta Z=5.0(N=5)$. For the values of effective parameters used while plotting graphs given on fig. 1 and 2 the critical value of the hydrogen bond length is $R_{\mathrm{OO}}^{c r}=2.36 \mathrm{~A}$ which is in accordance with the data known from literature (see $[5,10]$ ) for the cluster $\left(\mathrm{O}_{2} \mathrm{H}_{5}\right)^{+}$.

Similar graphs, obtained for cases $N=3$ and $N=5$ at the same values of the effective charges $Z_{i o n}$ and $Z_{e l}$ are given on fig. 3 and 4 . The shapes of dependences of the lowest energy levels versus $R_{\mathrm{OH}}$ qualitatively remains the same, as in the case of $N=4$, however, quantitative changes take place; $R_{\mathrm{OO}}^{c r}$ is equal now $2.33 \mathrm{~A}$ and $2.30 \mathrm{~A}$ correspondingly. The dependence of the critical length of the hydrogen bond $R_{\mathrm{O}}^{c r}$ on the number of the electrons on the cluster $\mathrm{O}-\mathrm{H}-\mathrm{O}$ is presented on fig. 5 .

At increasing of the hydrogen bond length the height of the potential barrier also increases as well as the $\delta$ value - the distance between positions of minima of the proton potential on the bond. The obtained relation between the $\delta$ parameter and the $R_{\mathrm{OO}}$ distance for different numbers of electrons on the cluster $\mathrm{O}-\mathrm{H}-\mathrm{O}$ is presented on fig. 6. This dependence becomes stronger when $R \rightarrow R_{\mathrm{OO}}^{c r}$, which is in agreement with results the known from literature (see for example [11]), good quantitative correlations also take place. It should be noted that the ratio $\Delta R_{\mathrm{HH}} / \Delta R_{\mathrm{OO}}$ at $R_{\mathrm{OO}} \geq R_{\mathrm{OO}}^{\text {cr }}$ is weakly sensitive to the change of the number of electrons on the bond.

\section{ELECTRON DENSITY DISTRIBUTION}

Using the eigenvectors of the Hamiltonian, corresponding, in particular, to the lowest eigenvalue $\lambda_{\text {min }}$, the distribution of the electron density can be obtained. The states of the basis of the diagonalized Hamiltonian are linearly expressed by the states of the previous basis

$$
|\tilde{i}\rangle=\sum_{j} \alpha_{i j}|j\rangle
$$

then the density of electron charge in the $|\tilde{i}\rangle$ state should be

$$
\tilde{\rho}_{i}(\mathbf{r})=\sum_{j} \alpha_{i j}^{2} \rho_{j}(\mathbf{r})
$$

The density of electron charge in the initial state $\rho_{j}(\mathbf{r})$, built on the basis of the wave functions $\left\{\varphi_{1}(\mathbf{r}), \varphi_{2}(\mathbf{r})\right.$, $\left.\varphi_{3}(\mathbf{r})\right\}$ can be expressed as

$$
\rho_{j}(\mathbf{r})=\sum_{i}^{3} n_{i}(j)\left|\varphi_{i}(\mathbf{r})\right|^{2}
$$

here $n_{i}(j)$ - occupation of the $\varphi_{i}(r)$ orbital in the $j$ initial state. For the $N=4$ case density distribution in the plane, in a which complex axis is located, is presented at fig. $7 \mathrm{a}$ and $7 \mathrm{~b}$ (for the proton located in one of the pots and in the middle of the bond correspondingly).

However, more informational is the distribution of electron density along the complex axis as a result of the integration of the space distribution over the planes, perpendicular to the complex axis, (see fig. 8a and 8b). The difference between the curves shows to us the redistribution of the electron density with the proton shifts from the central position.

On the basis of the electron density distribution in space the atomic orbitals occupations were calculated using the method of minimal squares. If $f(x)$ is obtained earlier electron distribution; and $F(x)=\sum_{i} n_{i} \psi_{i}^{2}(x)$ is some helper function (coefficients $n_{i}$ are occupations), then $n_{i}$ are found from the condition of the minimum of

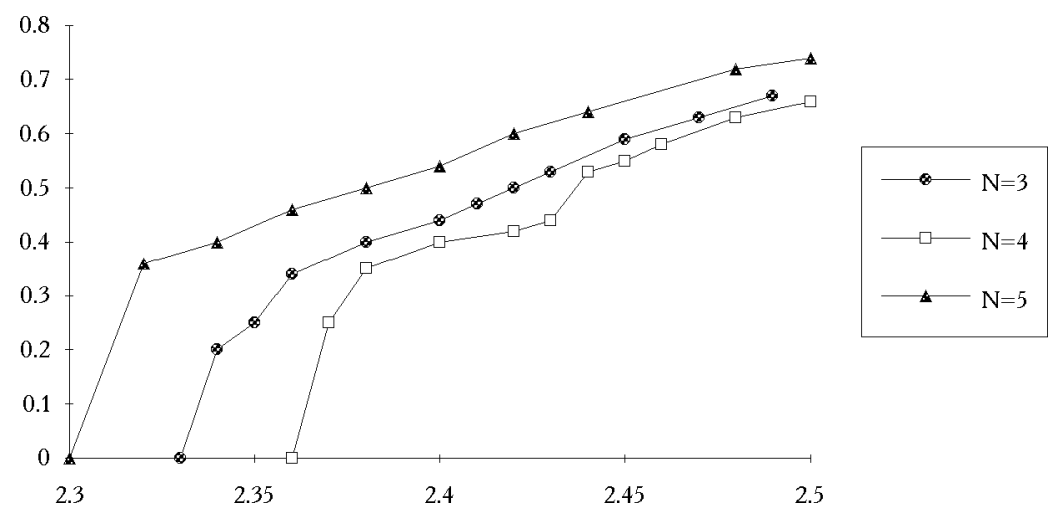

Fig. 6. Dependence of the distance between hydrogen potential minima $\delta$ on the complex length $R_{\odot \circ}$. 

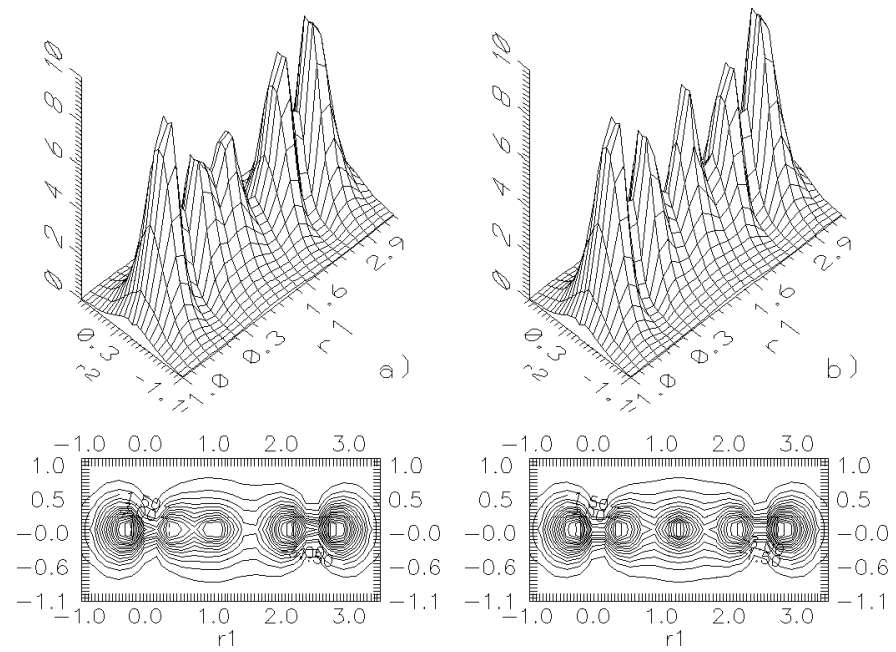

Fig. 7. Total electron density distribution in the plane containing a complex axis at different positions of the proton: a) in the left pot of the effective potential; b) in the middle of the bond. $N=4, Z_{e l}=0.5, Z_{\text {ion }}=4.5, R=2.40 \mathrm{~A}$

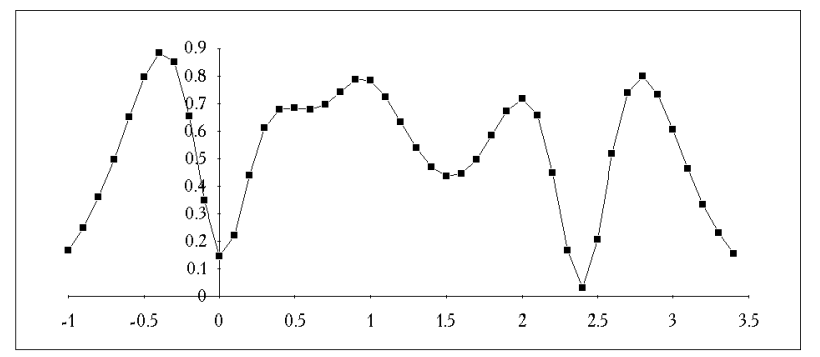

a)

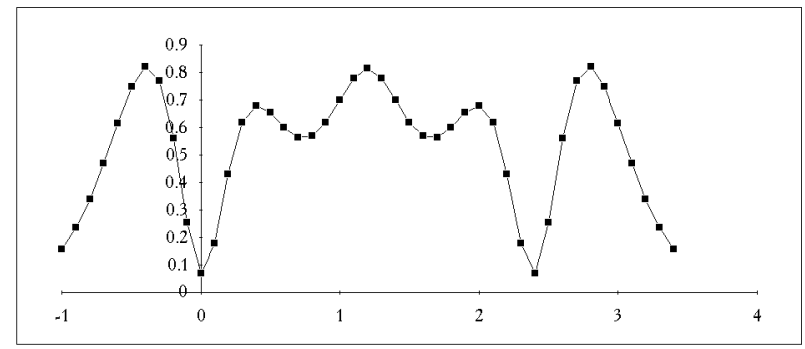

b)

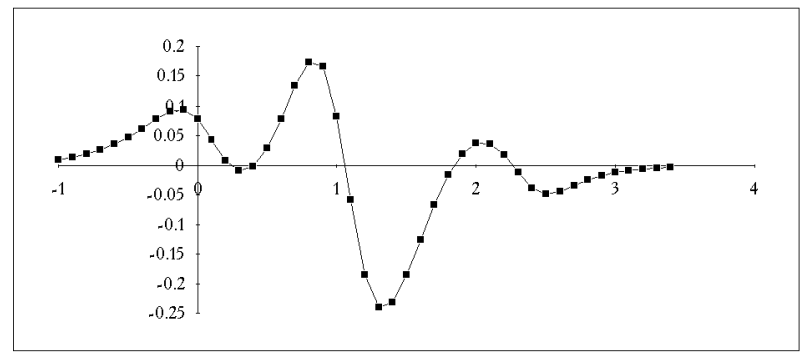

c)

Fig. 8. Linear electron density distribution along the complex axis at different positions of the proton: a) in the left pot of the effective potential; b) in the middle of the bond; c) change of electron density with the proton shifts from the central position (versus the value of the $R_{\mathrm{OH}}$ distance). $N=4, Z_{e l}=0.5, Z_{\text {ion }}=4.5, R=2.40 \mathrm{~A}$ 


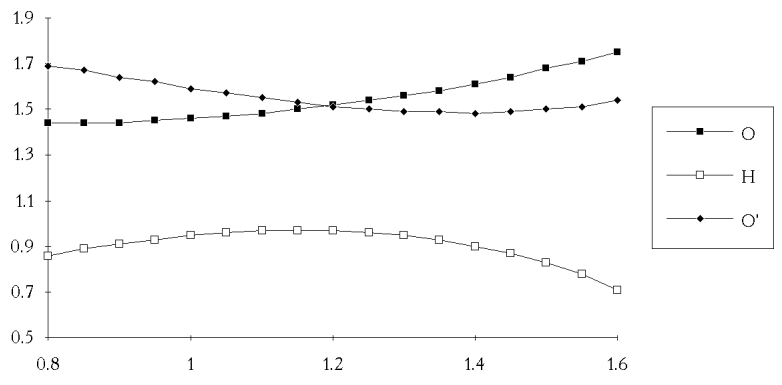

Fig. 9. Orbital occupation versus the $R_{\mathrm{OH}}$ distance $\left(N=4, Z_{e l}=0.5, Z_{i o n}=4.5, R=2.40 A\right)$. $O^{\prime}$ corresponds to the right oxygen ion.

$\sigma^{2}=\int(f(x)-F(x)) \psi_{i}^{2}(x)$. Solving the equation $\frac{\partial \sigma^{2}}{\partial n_{i}}=0$ we obtain orbital occupations (see fig. 9) as dependences on the $R_{\mathrm{OH}}$ distance.

A change of the occupation of the $2 p$ oxygen orbitals at the shift of the proton from one minimum into another is rather significant and approach to $0.2 \mathrm{e}$. This value corresponds to a change of the occupation of the $2 P_{z}$ oxygen orbitals point along the $\mathrm{H}$-bond in the complexes $\left(\mathrm{O}_{2} \mathrm{H}_{3}\right)^{-},\left(\mathrm{O}_{2} \mathrm{H}_{5}\right)^{+},\left(\mathrm{CH}_{3} \mathrm{OH}\right)_{2} \mathrm{H}^{+}$[10]. In general, proton shift on the bond is accompanied by increasing the summary electron density at the regions to where the proton is moved (see fig. 7,8 ); however, the occupancy of the $2 p$-orbitals of the corresponding oxygen ion is decreased. The occupancy of the $1 s$-orbitals of hydrogen ion changes weakly and possesses maximum at the location of proton in the middle of the bond (see fig. 9). The obtained results are in agreement with the experimentally obtained change of the electron charge density with the displacement of halogen ions and protons in halogen-bridged metal complexes and may confirm the applicability of the model of electron-proton correlation in a system with the hydrogen bonds [12].

\section{CONCLUSIONS}

A lot of papers were devoted to the calculation of electron spectrum and adiabatic potential of the proton on the bond for different structures with hydrogen bonds. Our goal was to concentrate more on the investigation of the electron density change caused by the ion shifts, in particular, by hydrogen motion on the bond. Similar calculations for the cluster with few hydrogen bonds will allow us to investigate the connection between the electron density change and proton-proton short-range interactions. Describing the system with hydrogen bonds using different models one should take into account such interactions. Until now these correlations were postulated and their nature was not sufficiently clear. The role of the electron subsystem there is evidently special because electron redistribution caused by the proton shifts between equilibrium positions on the neighbouring bonds lead to the additional interactions. That is why the study of electron density change with proton shifts is important.
A more detailed study of this problem will provide an opportunity to formulate more real models for the description of such objects.

\section{ACKNOWLEDGEMENTS}

The work has been supported in part by the INTASUkraine-95-0133 grant and by the Foundation for Fundamental Investigations of the Ukrainian Ministry of Science and Technology, project No 2.4/174.

\section{APPENDIX 1. MATRIX BLOCKS CORRESPONDING TO THE EXAMINED CASES.}

Case $\mathrm{N}=3$ :

$$
\begin{aligned}
& H(23,23)=2 \epsilon_{1}+\epsilon_{2}+2 K-Q+U, \\
& H(23,25)=2 L_{1}-L_{3}+t^{\prime}, \\
& H(23,27)=-\Phi-\Phi_{1}-t \\
& H(23,29)=-L_{2}-\Phi_{a}-t_{a a^{\prime}}, \\
& H(23,30)=L_{4}, \\
& H(23,32)=-L_{3}^{\prime}, \\
& H(23,34)=L_{2}-L_{4}+\Phi_{a}+t_{a a^{\prime}}, \\
& H(23,39)=-L_{3}, \\
& H(23,41)=Q_{a}, \\
& H(25,25)=2 \epsilon_{1}+\epsilon_{3}-Q_{a}+U+2 V, \\
& H(25,27)=-L_{4}, \\
& H(25,29)=-L_{3}^{\prime}, \\
& H(25,30)=L_{1}^{\prime}+\Phi+t \text {, } \\
& H(25,32)=-2 \Phi_{a}-t_{a a^{\prime}}, \\
& H(25,34)=-L_{1}^{\prime}+L_{3}^{\prime}-\Phi-t, \\
& H(25,39)=Q \text {, } \\
& H(25,41)=-L_{3}, \\
& H(27,27)=\epsilon_{1}+2 \epsilon_{2}+2 K-Q+U^{\prime}, \\
& H(27,29)=L_{1}+\Phi_{1}^{\prime}+t^{\prime}, \\
& H(27,30)=-L_{1}+L_{3}-\Phi_{1}^{\prime}-t^{\prime}, \\
& H(27,32)=Q^{\prime} \text {, } \\
& H(27,34)=-L_{3},
\end{aligned}
$$




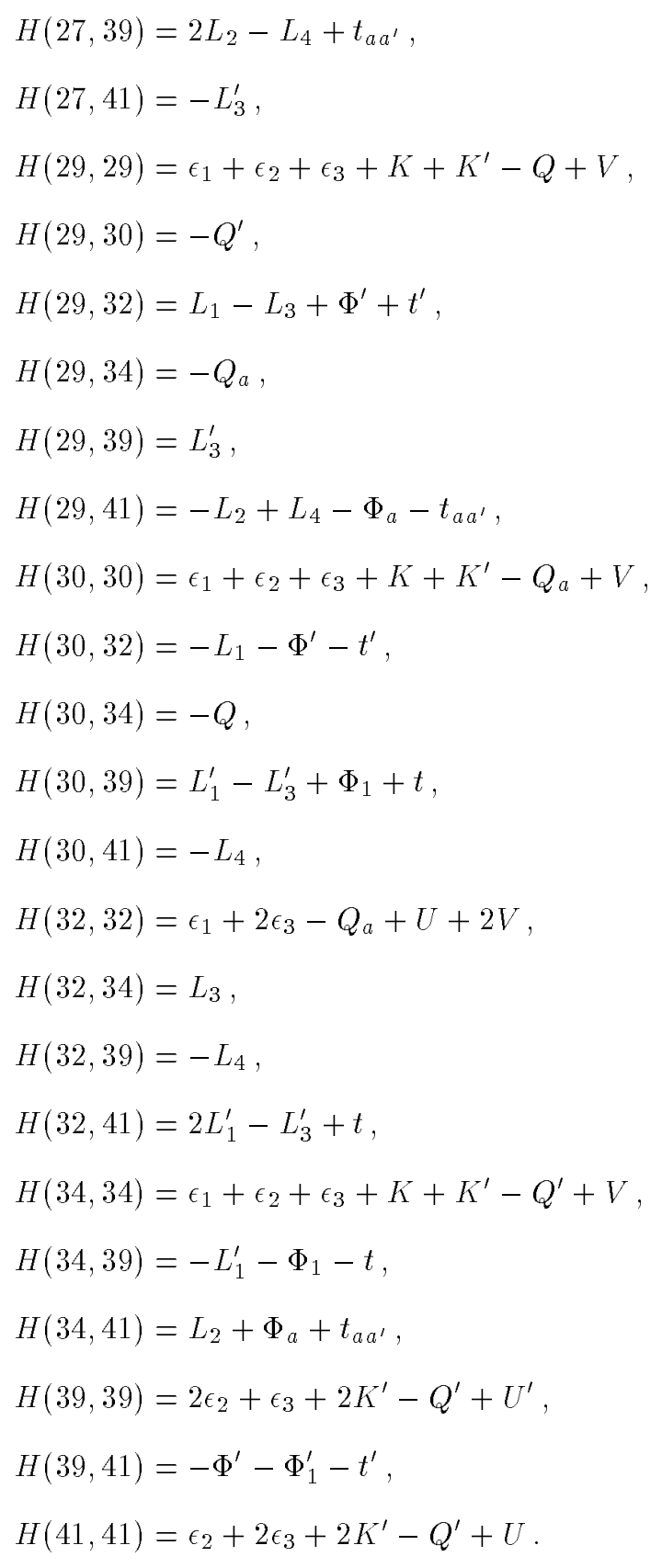

Case $\mathrm{N}=4$ :

$$
\begin{aligned}
& H(43,43)=2 \epsilon_{1}+2 \epsilon_{2}+4 K-2 Q+U+U^{\prime}, \\
& H(43,45)=2 L_{1}-L_{3}+\Phi_{1}^{\prime}+t^{\prime}, \\
& H(43,46)=-2 L_{1}+L_{3}-\Phi_{1}^{\prime}-t^{\prime}, \\
& H(43,48)=Q^{\prime} \\
& H(43,50)=2 L_{2}-L_{4}+\Phi_{a}+t_{a a^{\prime}}, \\
& H(43,52)=-L_{3}^{\prime} \\
& H(43,53)=-2 L_{2}+L_{4}-\Phi_{a}-t_{a a^{\prime}}, \\
& H(43,55)=L_{3}^{\prime},
\end{aligned}
$$

$$
\begin{aligned}
H(43,57) & =Q_{a}, \\
H(45,45) & =2 \epsilon_{1}+\epsilon_{2}+\epsilon_{3}+2 K+K^{\prime}-Q \\
& -Q_{a}+U+2 V, \\
H(45,46) & =-Q^{\prime}, \\
H(45,48) & =2 L_{1}-L_{3}+\Phi^{\prime}+t^{\prime}, \\
H(45,50) & =-L_{1}^{\prime}+L_{3}^{\prime}-\Phi-\Phi_{1}-t, \\
H(45,52) & =L_{4}, \\
H(45,53) & =-L_{3}^{\prime}, \\
H(45,55) & =L_{2}-L_{4}+2 \Phi_{a}+t_{a a^{\prime}}, \\
H(45,57) & =-L_{3}, \\
H(46,46) & =2 \epsilon_{1}+\epsilon_{2}+\epsilon_{3}+2 K+K^{\prime}-Q \\
& -Q_{a}+U+2 V,
\end{aligned}
$$

$H(46,48)=-2 L_{1}+L_{3}-\Phi^{\prime}-t^{\prime}$,

$H(46,50)=-L_{3}^{\prime}$,

$H(46,52)=L_{2}-L_{4}+2 \Phi_{a}+t_{a a^{\prime}}$,

$H(46,53)=-L_{1}^{\prime}+L_{3}^{\prime}-\Phi-\Phi_{1}-t$,

$H(46,55)=L_{4}$,

$H(46,57)=L_{3}$,

$H(48,48)=2 \epsilon_{1}+2 \epsilon_{3}-2 Q_{a}+2 U+4 V$.

$H(48,50)=-L_{4}$,

$H(48,52)=2 L_{1}^{\prime}-L_{3}^{\prime}+\Phi+t$,

$H(48,53)=L_{4}$,

$H(48,55)=-2 L_{1}^{\prime}+L_{3}^{\prime}-\Phi-t$,

$H(48,57)=Q$,

$H(50,50)=\epsilon_{1}+2 \epsilon_{2}+\epsilon_{3}+2 K+2 K^{\prime}-Q$

$$
-Q^{\prime}+U^{\prime}+V \text {, }
$$

$H(50,52)=-L_{1}+L_{3}-\Phi^{\prime}-\Phi_{1}^{\prime}-t^{\prime}$,

$H(50,53)=-Q_{a}$,

$H(50,55)=-L_{3}$,

$H(50,57)=2 L_{2}-L_{4}+\Phi_{a}+t_{a a^{\prime}}$,

$H(52,52)=\epsilon_{1}+\epsilon_{2}+2 \epsilon_{3}+K+2 K^{\prime}-Q_{a}$

$$
-Q^{\prime}+U+2 V \text {, }
$$




$$
\begin{aligned}
H(52,53) & =-L_{3}, \\
H(52,55) & =-Q, \\
H(52,57) & =2 L_{1}^{\prime}-L_{3}^{\prime}+\Phi_{1}+t, \\
H(53,53) & =\epsilon_{1}+2 \epsilon_{2}+\epsilon_{3}+2 K+2 K^{\prime}-Q \\
& -Q^{\prime}+U^{\prime}+V, \\
H(53,55) & =-L_{1}+L_{3}-\Phi^{\prime}-\Phi_{1}^{\prime}-t^{\prime}, \\
H(53,57) & =-2 L_{2}+L_{4}-\Phi_{a}-t_{a a^{\prime}}, \\
H(55,55) & =\epsilon_{1}+\epsilon_{2}+2 \epsilon_{3}+K+2 K^{\prime}-Q_{a} \\
& -Q^{\prime}+U+2 V, \\
H(55,57) & =-2 L_{1}^{\prime}+L_{3}^{\prime}-\Phi_{1}-t, \\
H(57,57) & =2 \epsilon_{2}+2 \epsilon_{3}+4 K^{\prime}-2 Q^{\prime}+U+U^{\prime} .
\end{aligned}
$$

Case $\mathrm{N}=5$ :

$$
\begin{aligned}
H(58,58) & =2 \epsilon_{1}+2 \epsilon_{2}+\epsilon_{3}+4 K+2 K^{\prime} \\
& -2 Q-Q_{a}-Q^{\prime}+U+U^{\prime}+2 V, \\
H(58,60) & =-2 L_{1}+L_{3}-\Phi^{\prime}-\Phi_{1}^{\prime}-t^{\prime}, \\
H(58,62) & =-2 L_{2}+L_{4}-2 \Phi_{a}-t_{a a^{\prime}}, \\
H(60,60) & =2 \epsilon_{1}+\epsilon_{2}+2 \epsilon_{3}+2 K+2 K^{\prime} \\
& -Q-2 Q_{a}-Q^{\prime}+2 U+4 V, \\
H(60,62) & =-2 L_{1}^{\prime}+L_{3}^{\prime}-\Phi-\Phi_{1}-t, \\
H(62,62) & =\epsilon_{1}+2 \epsilon_{2}+2 \epsilon_{3}+2 K+4 K^{\prime} \\
& -Q-Q_{a}-2 Q^{\prime}+U+U^{\prime}+2 V .
\end{aligned}
$$

\section{APPENDIX 2. SOME INTEGRALS USED IN THE ELECTRON SPECTRA OF THE O-H-O' CLUSTER CALCULATIONS.}

1. We have obtained analytical expressions for some integrals used for the calculations of the $\mathrm{O}-\mathrm{H}-\mathrm{O}^{\prime}$ cluster electron spectra. The atomic orbitals were chosen in the form of the Slater functions:

$$
\psi=R_{n l}(r) \cdot Y_{l m}(\theta, \varphi) .
$$

Radial part of the function has the following form:

$$
R_{n l}(\mathbf{r})=A_{n, l}(\alpha) r^{n^{*}-1} e^{-\alpha \frac{r}{a_{0}}},
$$

where the normalized factor $A_{n, l}(\alpha)$ is equal:

$$
\left[A_{n, l}(\alpha)\right]^{2}=\int_{0}^{\infty} d r r^{-2 n^{*}} e^{-2 \alpha \frac{r}{a_{0}}}, \alpha=\frac{z^{*}}{n^{*}},
$$

$n^{*}$ - effective quantum number (for $1 s, 2 p$-functions $\left.n^{*}=n\right), z^{*}$ - effective nuclear charge, $a_{0}$ - the first Bohr orbital radius. Generally the values $\alpha$ (or $z^{*}$ ) are the variational parameters but one can use the values for them obtained for this atom in other compounds (see Appendix 3).

We have calculated the two centre integrals using spheroidal coordinates $[13,14]$

$$
\lambda=\frac{r_{a}+r_{b}}{R}, \mu=\frac{r_{a}-r_{b}}{R}, \varphi=\varphi_{a}=\varphi_{b} .
$$

The element of the volume in these coordinates is:

$$
d V=\frac{R^{3}}{8}\left(\lambda^{2}-\mu^{2}\right) d \lambda d \mu d \varphi
$$

there are the boundaries of integration:

$$
1 \leq \lambda \leq \infty,-1 \leq \mu \leq 1,0 \leq \varphi \leq 2 \pi
$$

Here $r_{a}$ are $r_{b}$ mean the electron distances from the two centers $a$ and $b, \mathrm{R}$ is the distance between the centers.

We present the analytical expressions obtained for some molecular integrals which are not mentioned in the literature, for example in $[13,14]$ :

$$
\begin{aligned}
& \int \psi_{A}(\mathbf{r}) \frac{e^{2}}{r_{A}} \psi_{B}(\mathbf{r}) d \mathbf{r}=\frac{1}{4} \cdot \frac{e^{2}}{a_{0}} \alpha_{H} \alpha_{0}^{2} \sqrt{\frac{1}{3} \alpha_{H} \alpha_{0}}\left(\frac{R}{a_{0}}\right)^{3} \times \frac{1}{p} e^{-p} \cdot \frac{4}{\gamma}\left\{\frac{1}{p}\left(1+\frac{1}{p}\right) \sinh \gamma+\frac{1}{\gamma}\left(\cosh \gamma-\frac{1}{\gamma} \sinh \gamma\right)\right\} \\
& \int \psi_{A}(\mathbf{r}) \frac{e^{2}}{r_{B}} \psi_{B}(\mathbf{r}) d \mathbf{r}=\frac{1}{4} \cdot \frac{e^{2}}{a_{0}} \alpha_{H} \alpha_{0}^{2} \sqrt{\frac{1}{3} \alpha_{H} \alpha_{0}}\left(\frac{R}{a_{0}}\right)^{3} \times\left\{e^{\gamma}+\left(\frac{1}{p}-\frac{1}{\gamma}\right) \sinh \gamma\right\} .
\end{aligned}
$$

Here $\psi_{A}(\mathbf{r})-2 p$-oxygen orbital directed along the line connecting the centres $\left(2 P_{\sigma}\right), \psi_{B}(\mathbf{r})-1 s$-hydrogen orbital. Here: 


$$
p=\frac{\alpha_{b}+\alpha_{A}}{2} \cdot \frac{R}{a_{0}}, \quad \gamma=\frac{\alpha_{b}-\alpha_{A}}{2} \cdot \frac{R}{a_{0}} .
$$

We have obtained the energy of the Coulomb repulsion between the two electrons in the $1 s$-state of the hydrogen atom:

$$
\int \psi_{B}^{2}\left(\mathbf{r}_{1}\right) \frac{e^{2}}{r_{12}} \psi_{B}^{2}\left(\mathbf{r}_{2}\right) d \mathbf{r}_{1} d \mathbf{r}_{2}=\frac{5}{8} \cdot \frac{e^{2}}{a_{0}} \mu_{H}
$$

which coincides with the expression for the Helium atom known in the literature [13]. In the case of the Coulomb repulsion between the two electrons in the $2 p$-state of the oxygen atom we have:

$$
\int \psi_{\boldsymbol{A}}^{2}\left(\mathbf{r}_{\mathbf{1}}\right) \frac{e^{2}}{r_{12}} \psi_{A}^{2}\left(\mathbf{r}_{\mathbf{2}}\right) d \mathbf{r}_{\mathbf{1}} d \mathbf{r}_{\mathbf{2}}=\frac{501}{1280} \cdot \frac{e^{2}}{a_{0}} \mu_{0} .
$$

Here $\left|\mathbf{r}_{1}-\mathbf{r}_{\mathbf{2}}\right|=r_{12}$. Below we present the formula obtained for one of the hybrid integrals:

$$
\begin{aligned}
& \int \psi_{B}^{2}\left(\mathbf{r}_{1}\right) \frac{e^{2}}{r_{12}} \psi_{\boldsymbol{A}}\left(\mathbf{r}_{2}\right) \psi_{B}\left(\mathbf{r}_{2}\right) d \mathbf{r}_{1} d \mathbf{r}_{2} \\
& =\frac{1}{4} \frac{e^{2}}{a_{0}}\left(\alpha_{H}\right)^{3 / 2}\left(\alpha_{0}\right)^{5 / 2}\left(\frac{R}{a_{0}}\right)^{3} \cdot\left(\frac { 4 } { \gamma } \cdot \frac { 1 } { p } e ^ { - p } \left\{\left(1+\frac{1}{p}\right) \cdot\left(\sinh \gamma-\frac{1}{\gamma} \cosh \gamma+\frac{1}{\gamma^{2}} \sinh \gamma\right)\right.\right. \\
& \left.-\left(1+\frac{1}{p_{1}}+\frac{1}{p_{1}^{2}}\right)\left(-\cosh \gamma_{1}+\frac{1}{\gamma_{1}} \sinh \gamma_{1}\right)\right\}-\frac{4}{\gamma_{1}} \cdot \frac{1}{p_{1}} e^{-p_{1}}\left\{\left(1+\frac{1}{p_{1}}\right)\left(\sinh \gamma_{1}-\frac{\cosh \gamma_{1}}{\gamma_{1}}+\frac{\sinh \gamma_{1}}{\gamma_{1}^{2}}\right)\right. \\
& \left.-\left(1+\frac{1}{p_{1}}+\frac{1}{p_{1}^{2}}\right)\left(-\cosh \gamma_{1}+\frac{\sinh \gamma_{1}}{\gamma_{1}}\right)\right\}-\frac{\alpha_{H}}{2} \frac{R}{a_{0}} \frac{4}{\gamma_{1}} \frac{1}{p_{1}} e^{-p_{1}}\left\{\left(\frac{1}{p_{1}}+\frac{1}{\gamma_{1}}\right) e^{\gamma_{1}}+\left(\frac{1}{p_{1}^{2}}+\frac{1}{\gamma_{1}^{2}}\right) \sinh \gamma_{1}\right. \\
& \left.\left.-3\left(\frac{1}{p_{1}^{2}}+\frac{1}{p_{1}^{3}}\right)\left(-\cosh \gamma_{1}+\frac{1}{\gamma_{1}} \sinh \gamma_{1}\right)++3\left(1+\frac{1}{p_{1}}\right)\left(-\frac{1}{\gamma_{1}^{2}} \cosh \gamma_{1}+\frac{1}{\gamma_{1}^{3}} \sinh \gamma_{1}\right)\right\}\right),
\end{aligned}
$$

where

$$
p_{1}=\frac{3 \alpha_{H}+\alpha_{0}}{2} \cdot \frac{R}{a_{0}} ; \quad \gamma_{1}=\frac{3 \alpha_{H}-\alpha_{0}}{2} \cdot \frac{R}{a_{0}} .
$$

2. One can obtain the analytical expression for the exchange integral

$$
\tilde{Q}=\int \psi_{A}^{*}\left(\mathbf{r}_{1}\right) \psi_{B}^{*}\left(\mathbf{r}_{\mathbf{2}}\right) \cdot \frac{e^{2}}{\left|\mathbf{r}_{\mathbf{1}}-\mathbf{r}_{\mathbf{2}}\right|} \psi_{B}\left(\mathbf{r}_{\mathbf{1}}\right) \psi_{A}\left(\mathbf{r}_{\mathbf{2}}\right) d \mathbf{r}_{\mathbf{1}} d \mathbf{r}_{\mathbf{2}}
$$

only in the case of the hydrogen molecule, or in the case where $\psi_{A}\left(\mathbf{r}_{1}\right)$ and $\psi_{B}\left(\mathbf{r}_{2}\right)$ are the same $n S$ functions which are centered at $A$ and $B$ points. In the other cases one can obtain the analytical expression for the exchange integral only in the form of the infinite series. The authors propose the general scheme of the calculation and the formula in the form of the infinity series obtained for the exchange integrals in the case when the electron functions $\psi(\mathbf{r})$ are the $S$-type functions $n_{1} S$ and $P$-type functions directed along the line connecting the atoms $\left(n_{2} P_{\sigma}\right) ; n_{1}, n_{2}-$ the principal quantum numbers. We have used the expansion into a series in the spheroidal coordinates of the $\frac{1}{\left|\mathbf{r}_{1}-\mathbf{r}_{2}\right|}$ function [14]:

$$
\frac{1}{\left|\mathbf{r}_{1}-\mathbf{r}_{2}\right|}=\frac{2}{R} \sum_{k=0}^{\infty} \sum_{m=-k}^{k}(-1)^{m}(2 k+1)\left[\frac{(k-|m|) !}{(k+|m|) !}\right]^{2} P_{k}^{|m|}[\lambda(a)] * Q_{k}^{|m|}[\lambda(b)] P_{k}^{|m|}\left(\mu_{1}\right) P_{k}^{|m|}\left(\mu_{2}\right) e^{i m\left(\varphi_{1}-\varphi_{2}\right)}
$$

Here $\lambda_{1}, \mu_{1}, \varphi_{1}$ and $\lambda_{2}, \mu_{2}, \varphi_{2}$ - spheroidal coordinates of the two electrons, $\lambda(a)$ - is the minimum and $\lambda(b)-$ is the maximum from $\lambda_{1}$ and $\lambda_{2}, Q_{k}^{|m|}$ - the Legendre functions of the second type. 


$$
\begin{aligned}
& Q_{k}^{|m|}(x)=\left(1-x^{2}\right)^{\frac{|m|}{2}} \frac{d^{|m|}}{d x^{m}} Q_{k}(x) \\
& Q_{k}(x)=\frac{1}{2} P_{k}(x) \ln \frac{x+1}{x-1}-\frac{1 \cdot 3 \ldots(2 k-1)}{k !} \cdot\left\{x^{k-1}+x^{k-3}\left[\frac{1}{3}-\frac{k(k-1)}{2(2 k-1)}\right]\right. \\
& \left.+x^{k-5} \cdot\left[\frac{1}{5}-\frac{1}{3} \frac{k(k-1)}{2(2 k-1)}+\frac{k(k-1)(k-2)(k-3)}{2 \cdot 4(2 k-1)(2 k-3)}\right]+\ldots\right\} .
\end{aligned}
$$

A series continues to the last positive power of $x$.

Quantum numbers $m_{1}$ and $m_{2}$ equal zero for the $n_{1} S$ - and $n_{2} P_{\sigma}$-functions therefore the only components $m=0$ from the sum over $m$ in (27) give a contribution to $\tilde{Q}(26)$ which will be:

$$
\frac{2}{R} \sum_{k=0}^{\infty}(2 k+1) P_{k}[\lambda(a)] Q_{k}[\lambda(b)] P_{k}\left(\mu_{1}\right) P_{k}\left(\mu_{2}\right) .
$$

Then we go to the spheroidal coordinates in the integrand for $\tilde{Q}$ (26). Let us consider the part of the integrand (without $\frac{1}{r_{12}}$ ) depending on one variable $\psi_{A}\left(\mathbf{r}_{\mathbf{1}}\right) \psi_{B}\left(\mathbf{r}_{\mathbf{1}}\right) d \mathbf{r}_{\mathbf{1}}$. After transition to the spheroidal coordinates we obtain:

$$
\psi_{A}\left(\mathbf{r}_{1}\right) \psi_{B}\left(\mathbf{r}_{1}\right) d \mathbf{r}_{1}=\beta M_{1}\left(\lambda_{1}, \mu_{1}\right),
$$

where $M_{1}\left(\lambda_{1}, \mu_{1}\right)$ is a polynomial in the spheroidal coordinates.

The coefficient $\beta$ does not involve the variable of integration and is equal to the product of normalized factors of the functions $\psi_{A}$ and $\psi_{B}$; also it includes the multiplier $2 \pi\left(\frac{R}{2}\right)^{n_{1}+n_{2}+1}$. The coefficient $\beta$ equals $\beta=2\left(\frac{\alpha_{H}}{a_{0}}\right)^{3 / 2}\left(\frac{\alpha_{n}}{a_{0}}\right)^{5 / 2}\left(\frac{R}{2}\right)^{4}$ in the case when $\psi_{A}=2 \rho_{\sigma}(0)$, $\psi_{B}=1 S(H)$. Let us consider the expression under the integral in $\lambda_{1}, \mu_{1}$ variables for the $k$-term of the series. Expression (29) is multiplied by the Legendre polynomial $P_{k}\left(\mu_{1}\right)$ :

$$
\beta M_{1}\left(\lambda_{1}, \mu_{1}\right) * P_{k}\left(\mu_{1}\right)=\beta M_{2}^{k}\left(\lambda_{1}, \mu_{1}\right) .
$$

Now, the variable $\mu_{1}$ is involved in the $M_{2}^{k}$ polynomial. The integration of the $M_{2}^{k}\left(\lambda_{1}, \mu_{1}\right)$ polynomial over the $\mu_{1}$ variable is not complicated:

$$
\int_{-1}^{1} \mu_{1}^{n} e^{-\gamma \mu_{1}} d \mu_{1}=F(n, \gamma, 1)-F(n, \gamma,-1)
$$

where

$$
F(n, \alpha, r) \equiv \int r^{n} e^{-\alpha r} d r=-\frac{n !}{\alpha^{n+1}} e^{-\alpha r} \sum_{s=0}^{n} \frac{(\alpha r)^{s}}{s !}
$$

Denote

$$
\beta \int_{-1}^{1} M_{2}^{k}\left(\lambda_{1}, \mu_{1}\right) d \mu_{1}=\beta M_{3}^{k}\left(\lambda_{1}\right)
$$

The obtained polynomial in the variable $\lambda_{1}(33)$ is multiplied further by the Legendre function of the $\lambda_{1}$ variable from series (27). Denote

$$
\beta M_{3}^{k}\left(\lambda_{1}\right) \cdot P_{k}\left(\lambda_{1}\right)=\beta \sum_{i} A_{i}^{k} \lambda_{i}^{m_{i}}
$$

In the integrand for $\tilde{Q}$ the following components are also presented:

$$
\beta M_{3}^{k}\left(\lambda_{1}\right) * f_{k}\left(\lambda_{1}\right)=\beta \sum_{j} C_{j}^{k} \lambda_{i}^{m_{j}}
$$

We write (see 28)

$$
Q_{k}(x)=\frac{1}{2} P_{k}(x) \ln \frac{x+1}{x-1}-f_{k}(x) .
$$

We have obtained the general formula for the exchange integral (26)

$$
\begin{aligned}
\tilde{Q} & =\frac{e^{2}}{R} \beta^{2} \sum_{k=0}^{\infty}(2 k+1) \sum_{i} \sum_{j}\left(A _ { i } ^ { k } A _ { j } ^ { k } \left\{F\left(m_{i}, p, 1\right) \cdot F\left(m_{j}, p, 1\right)[c+\ln p]\right.\right. \\
& -\left[F\left(m_{i}, p,-1\right) F\left(m_{j}, p, 1\right)+F\left(m_{i}, p, 1\right) F\left(m_{j}, p,-1\right)\right] \times E_{i}(-2 p)+F\left(m_{i}, p,-1\right) F\left(m_{j}, p,-1\right) * E_{i}(-4 p)
\end{aligned}
$$




$$
\begin{aligned}
& +F\left(m_{i}, p, 1\right) \cdot \frac{m_{j} !}{p^{m_{j}+1}} e^{-p} \sum_{s=1}^{m_{j}} \frac{p^{s}}{s !} \sum_{k^{\prime}=0}^{s-1} C_{s}^{k^{\prime}} \frac{\left(s-k^{\prime}-1\right) !}{p^{s-k^{\prime}}} \times\left(1-\frac{1}{2^{s-k^{\prime}}}-(-1)^{k^{\prime}} \sum_{l=0}^{s-k^{\prime}-1} \frac{(2 p)^{l}}{l !}\right) \\
& -F\left(m_{i}, p,-1\right) \cdot \frac{m_{j} !}{p^{m_{j}+1}} e^{p} \sum_{s=1}^{m_{j}} \frac{p^{s}}{s !} \sum_{k^{\prime}=0}^{s-1}(-1)^{k^{\prime}} C_{s}^{k^{\prime}} \cdot F\left(s-k^{\prime}-1,2 p, 2\right) \\
& \left.+\frac{m_{i} !}{p^{m_{i}+1}} \sum_{s=1}^{m_{i}} \frac{p^{s}}{s !} \sum_{k^{\prime}=0}^{s-1} C_{s}^{k^{\prime}} \frac{\left(s-k^{\prime}-1\right) !}{p^{s-k^{\prime}}} \times \sum_{l=0}^{s-k^{\prime}-1} \frac{p^{l}}{l !} \sum_{l_{1}=0}^{l} C_{l}^{l_{1}} F\left(m_{j}+l-l_{1}, 2 p, 1\right) \cdot\left[(-1)^{l_{1}}-(-1)^{k^{\prime}}\right]\right\} \\
& +2 A_{i}^{k} C_{j}^{k}\left\{-\frac{m_{i} !}{p^{m_{i}+1}} \sum_{s=0}^{m_{i}} \frac{p^{s}}{s !} F\left(n_{j}+s, 2 p, 1\right)-F\left(m_{i}, p, 1\right) \cdot F\left(n_{j}, p, 1\right)+\frac{n_{j} !}{p^{n_{j}+1}} \sum_{l=0}^{n_{j}} \frac{p^{l}}{l !} F\left(m_{i}+l, 2 p, 1\right)\right\},
\end{aligned}
$$

$E_{i}(x)$ is the exponential integral

$$
E_{i}(-x)=-\int_{x}^{\infty} \frac{e^{-t}}{t} d t, \text { where } x>0
$$

$c$ is the Euler constant

$$
c=\int_{0}^{1} \frac{1-e^{-t}}{t} d t-\int_{1}^{\infty} \frac{e^{-t}}{t} d t=0.577216,
$$

$C_{s}^{k}=\frac{s !}{k !(s-k) !}-$ coefficient from the Newton binomial.

Let us consider the example of the described scheme realization in the calculation of the exchange integrals for the hydrogen molecule. We have

$$
M_{1}=\lambda_{1}^{2}-\mu_{1}^{2}, \beta=\frac{1}{4}\left(\alpha \frac{R}{a_{0}}\right)^{3}, \alpha=1 .
$$

The integration over the variable $\mu_{1}$ produces only two nonzero terms of series (27): $k=0$ and $k=2$. We have for $k=0$ :

$$
M_{2}^{(0)}\left(\lambda_{1}, \mu_{1}\right)=M_{1}\left(\lambda_{1}, \mu_{1}\right) .
$$

In accordance with (33) $M_{3}^{(0)}\left(\lambda_{1}\right)=2 \lambda_{1}^{2}-2 / 3$ and $A_{1}^{(0)}=2, m_{1}=2 ; A_{2}^{(0)}=-2 / 3, m_{2}=0$.

We have for $k=2$ :

$$
M_{2}^{(2)}\left(\lambda_{1}, \mu_{1}\right)=P_{2}\left(\mu_{1}\right)\left(\lambda_{1}^{2}-\mu_{1}^{2}\right),
$$

$$
\begin{aligned}
& M_{3}^{(2)}\left(\lambda_{1}\right)=-4 / 5 \\
& \sum_{i} A_{i}^{(2)} \lambda_{i}^{m_{i}}=-\frac{4}{5} P_{2}\left(\lambda_{1}\right)=-\frac{2}{15}\left(2 \lambda_{1}^{2}-1\right),
\end{aligned}
$$

therefore $A_{1}^{(2)}=-2 / 5, m_{1}=2 ; A_{2}^{(2)}=2 / 15, m_{2}=0$.

Also $\sum_{j} C_{j}^{(2)} \lambda_{1}^{n_{j}}=M_{3}^{(2)}\left(\lambda_{1}\right) f_{2}\left(\lambda_{1}\right)=-(2 / 5) \lambda_{1}$ and $C_{1}^{(2)}=-2 / 5, n_{1}=1$.

One can obtain the known formula for the exchange integral of the hydrogen molecule [14] using expression (37) and using the obtained above nonzero coefficients $A_{i}, C_{j}$.

\section{APPENDIX 3. EFFECTIVE PARAMETERS OF THE SLATER TYPE ATOMIC ORBITALS.}

It was obtained on the basis of a considerable amount of results of the electron structure calculations of the molecules and clusters that the behaviour of a certain atom in some class of compounds is similar and the calculations of the spectra of these compounds or molecules in them give us similar values of the effective parameters (which were taken as variational parameters). The rules of the calculations of the effective parameters of the Slater type atomic orbitals on the basis of these results are determined $[15,16,17]$. We used the modified SlaterEngus rules in the calculations of the effective parameters $Z^{*}$ and $n^{*}$ [17]. In compliance with them we have for $n \leq 3, n^{*}=n$ and for the hydrogen and oxygen atom:

$$
\begin{aligned}
& \psi_{1 s}^{(H)}: Z^{*}=1, n^{*}=1, \alpha\left(1 S_{H}\right)=1.0, \\
& \psi_{2 p}^{(O)}: Z^{*}=4.55, n^{*}=2, \alpha\left(2 P_{O}\right)=2.275 .
\end{aligned}
$$

One can take into account the ionic state of the oxygen atom in the compounds, for example for $O^{-1}$ and $O^{-1.5}$ we have

$$
\begin{aligned}
& \psi_{2 p}^{\left(O^{-1}\right)}: Z^{*}=4.2, n^{*}=2, \alpha\left(2 P_{O^{-1}}\right)=2.1, \\
& \psi_{2 p}^{\left(O^{-1.5}\right)}: Z^{*}=4.025, n^{*}=2, \alpha\left(2 P_{O^{-1.5}}\right)=2.012 .
\end{aligned}
$$


[1] S. Scheiner, in Reviews in Computational Chemistry 2. ch.6, edited by K. B. Lipkowitz, D. B. Boyd (VCH, New York, 1991), p. 165.

[2] S. Scheiner, in Aggregation Processes in Solution, edited by E. Wyn-Jones, J. Gorally (Elsevier, Amsterdam, 1983)

[3] The Hydrogen Bond. Recent Developments in Theory and Experiments, edited by P. Schuster, G. Zundel, C. Sandorfy (North-Holland, Amsterdam, 1976).

[4] Structure and Dynamics of Weakly Bound Molecular Complexes, edited by A. Weber (Reidel, Dordrecht, 1987).

[5] S. Scheiner, in Proton Transfer in Hydrogen-Bonded Systems, edited by T. Bountic (Plenum Press, New York, 1992).

[6] H. Sugimoto, S. Ikeda, Phys. Rev. Lett. 67, 1306 (1991)

[7] K. Okaniwa, H. Okamoto, T. Mitani, K. Toriumi, M. Yamashita, J. Phys. Soc. Jpn. 60, 997 (1991).

[8] J. Mustre de Leon, S. D. Conradson, I. Batistic, A. R. Bishop, Phys. Rev. B 44, 2422 (1991).

[9] I. V. Stasyuk, Yu. V. Sizonenko, Cond. Matt. Phys. (Lviv) iss. 5, 161 (1995).

[10] E. A. Hillenbrand, S. Scheiner, J. Amer. Chem. Soc. 106, 6266 (1984)
[11] E. Matsushita, T. Matsubara, Prog. Theor. Phys. 67, 1 (1982).

[12] E. Matsushita, Phys. Rev. B 51, 17332 (1995).

[13] P. Gombas, Theorie and Losungsmethoden des Mehrtielchenproblems der Wellenmechanic (Basel, 1950) [Translated into Russian (Inostrannaia Litieratura, Moscow, 1953)].

[14] J. Slater, Quantum Theory of Molecules and Solids. Volume 1. Electronic Structure of Molecules (Mc. Grawhill Book Company, Inc. New York - San Francisco - Toronto - London, 1963) [Translated into Russian (Mir, Moscow, 1965)].

[15] W. H. Flygare, Stroieniie i dinamika moliekul, t. 1 , t. 2 (Molecular structure and dynamics, v.1, v.2) (Mir, Moscow, 1982).

[16] I. B. Bersuker, Eliektronnoie stroienie i svoistva koordinatsionnykh soiedinienii (Electronic structure and properties of coordinational units) (Khimiia, Leningrad, 1986).

[17] S. S. Batsanov, P. A. Zviagina, Integraly pieriekryvaniia i probliema effiektivnykh zariadov, $t .1$ (Overlap integrals and problem of effective charges, v. 1) (Nauka, Novosibirsk, 1966).

\section{ЕЛЕКТРОННІ СТАНИ ТА АДІЯБАТИЧНІ ПОТЕНЦІЯЛИ ВОДНЕВОГО ЗВ’ЯЗКУ}

І. В. Стасюк, Ю. В. Сизоненко, Р. Я. Стеців

Інститут фізики конденсованих систем НАН Украйни,

вул. Свєнціцького, 1, Львів, 290011, Украйна

Вивчено властивості водневого зв'язку на прикладі найпростішого об'єкта, що містить водневий зв'язок, — йонного комплексу O-H-O. Отримано електронний енергетичний спектр комплексу O-H-О. Досліджено формування двомінімумного адіябатичногопотенціялу для протона зале жно від дов жини водневого зв'язку $R_{\circ \circ}$, відстані $R_{\odot \mathrm{H}}$, числа електронів $N$ на комплексі. Отримано карти електронної густини й розподілу електронного заряду вздов ж оси комплексу. Досліджено також зміну густини електронного заряду й заселеностей атомних орбіталей вихідного базису зале жно від змішення протона вздовж водневого зв'язку. 